\title{
Refractory Thrombocytopenia Associated to Disseminated Histoplasmosis in a Living-Related Renal Transplant Recipient: A Case Report and Review of the Literature
}

\author{
Rosario MD', Alfredo G-G ${ }^{1}$, De J esus $\mathrm{AOA}^{2}$ and \\ J orge $A^{1,2 *}$ \\ 'Department of Nephrology and Organ Transplant Unit, \\ Specialties Hospital, National Western Medical Centre, \\ Mexican Institute of Social Security, Mexico \\ ${ }^{2}$ Department of Physiology, University Health Sciences \\ Center, University of Guadalajara, Guadalajara, J alisco, \\ Mexico \\ *Correspondling author: Andrade Sierra J orge, \\ Department of Physiology, University Health Sciences \\ Center, University of Guadalajara, Guadalajara, J alisco, \\ Mexico
}

Received: J anuary 20, 2021; Accepted: J une 01, 2021; Published: J une 08, 2021

\section{Background}

Histoplasmosis (HP) is a systemic fungal infection, and the spectrum of the illness varies from asymptomatic primary infection to Disseminated Histoplasmosis (DH) in immunocompromised patients. Approximately $10 \%$ of infected patients can develop DH,and more so in states of sub-immunosuppression (organ transplant and/ or hematologic malignancies) [1]. Histoplasmosis after Solid Organ Transplantation (SOT) is not frequent, even in patients who live in endemic areas. The incidence of DH in SOT is less than 1\% [2] and can compromise the Medulla Ossium (MO) in up to one third of cases, with anemia and leukopenia as the most common alterations [3]. Thrombocytopenia is also reported in DH [4,5]; however, refractory cases have only been documented in immunocompetent patients [6], and there is no existing information on the immunocompromised. Recuperation of the depletion of cell lines occurs in a few weeks and is associated with the response to antifungal treatment $[7,8]$. The persistence of thrombocytopenia despite medical treatment is not common, and little information exists in immunocompromised patients. We present the first case in Mexico of DH in a renal transplant recipient (RTR) with a case of refractory thrombocytopenia.

\section{Past Medical History}

This is the case of a single, 30year-old, male resident of Guadalajara, Jalisco, Mexico, with a history of chronic kidney disease of unknown etiology, treated with hemodialysis for two years, and a RTR from a living-related donor (08/11/2019); with blood group compatibility, a haplotype of HLA, and cross-matched negative by flow cytometry. The serologic status for Cytomegalovirus (CMV) was Donor IgG+/ Receptor IgG+. The patient refused transfusions of hemoderivatives pre- and post-transplant. Induction immunosuppression was based inbasiliximab $20 \mathrm{mgs}$ (days 0 and 4 post-transplant), Tacrolimus
(TAC) dose of $0.12 \mathrm{mg} / \mathrm{kg} /$ day, Mycophenolate Mofetil (MMF) with a dose of $2 \mathrm{~g}$, and methylprednisolone of 500mgs. Maintenance immunosuppression was with TAC: dose of $4 \mathrm{mg} /$ day, MMF: dose of $2 \mathrm{~g} /$ day, and Prednisone (PDN): dose of $5 \mathrm{mg} /$ day. The antibacterial prophylaxis was comprised of trimethoprim/sulfamethoxazole $400 / 80 \mathrm{mg} /$ day for 6 months, and antiviral prophylaxis was not used. Serum Creatinine (SCr) at post-transplant discharge was $1.3 \mathrm{mg} / \mathrm{dL}$, without any clinical events of acute rejection; and, biopsies of the renal graft were not done in follow-up. The patient's history of a CMV infection three months prior was treated with Valgancyclovir (VGC) $900 \mathrm{mg}$ two times daily for 21 days followed by $450 \mathrm{mg}$ every 12 hours without suspension; and a polymerase chain reaction (PCR)for CMV $<150$ copies/mL was performed at 2 months of treatment.

\section{Diagnosis and Clinical Course of the Present Disease}

History of fever for four weeks with no response to antipyretics, for which the patient required hospitalization by his treating physician. Upon hospitalization (18/05/2020), the patient presented with hyporexia, nausea, diarrhea, and a fever of $39.5^{\circ} \mathrm{C}$. Physical examination showed an arterial pressure of130/80, normal oropharynx, no lymphadenopathies, no apparent respiratory and/or cardiovascular alterations, and no clinical presence of visceromegalies. Acute failure of the renal graft was documented with SCr $3.14 \mathrm{mg} / \mathrm{dL}$ and supra-optimal levels of TAC $17.6 \mathrm{ng} / \mathrm{mL}$ (Table 1). The patient received intensive hydration with crystalloid solutions and empirical antimicrobial management with levofloxacin, as well as modification of the immunosuppressionregimen (MMF $500 \mathrm{mg} /$ day and TAC 2 $\mathrm{mg} /$ day).Throughout follow-up, the ultrasound of the renal graft did not show any structural alterations, and the resistance indices were normal. Transthoracic echocardiogram was without vegetations, 


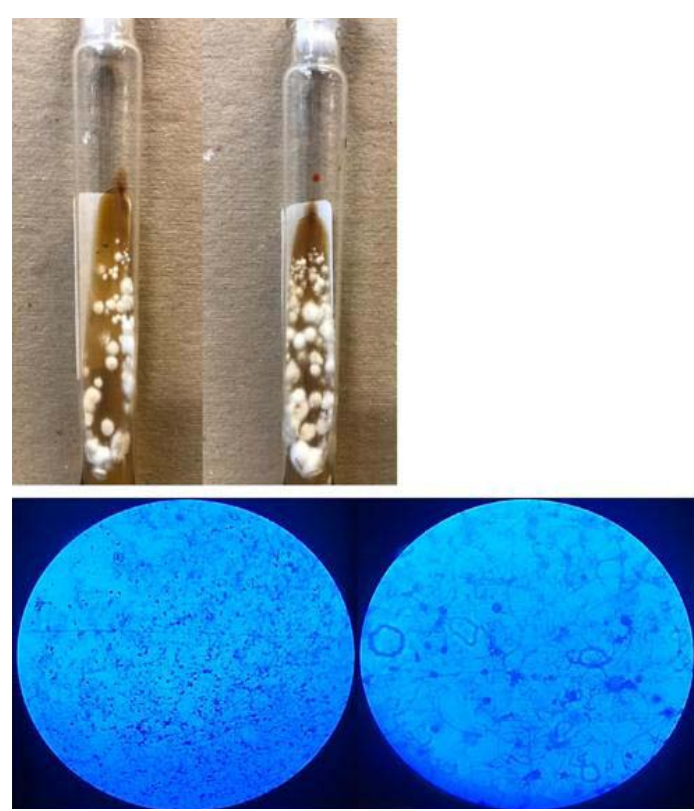

Figure 1: Macroscopic and microscopic appearance of Histoplasma sp. A) Macroscopic appearance of Histoplasma sp colonies, white and cottony. B) Microscopic appearance of Histoplasma $s p$, 10X image on the right and 40X image on the left. Thin, hyaline, and septate hyphae are seen, as well as macro and microconidia.

and the Left Ventricle Ejection Fraction (LVEF) was 65\%, without alterations in the segmental mobility. In the thoracic, abdominal, and pelvic tomography there were multiple inflamed lymph nodes of $12 \mathrm{~mm}$ in the mediastinum and $6 \mathrm{~mm}$ para-aortic and aortocaval nodes.

Day 1: Patient presented with spontaneous epistaxis associated with arterial pressure in the range of $160 / 100 \mathrm{mmHg}$ for which he required management with oral nifedipine $30 \mathrm{mg}$ every 12 hours, and prior management using tamponade. Day 3: Persistent fever of $40^{\circ} \mathrm{C}$ with negative blood culture and urine culture results. Empirical management was initiated with piperacillin/tazobactam at a dose of 4.5 gIV every 8 hours, DOTBAL (isoniazid 75, rifampicin 150, pirazinamide 400, ethambutol $300 \mathrm{mg}) 2$ tablets orally every 12 hours, and amphotericin B desoxycholate $25 \mathrm{mg}$ IV every 8 hours. Day 8: Persistent fever of $39^{\circ} \mathrm{C}$ and severe thrombocytopenia of 18 thousand $/ \mathrm{mm}^{3}$ without signs of bleeding. A bone Marrow (MO) aspirate and white blood cell cultures were performed. The aspirate was suggestive of an unspecific infectious process: [4] megakaryocytes/field; $14 \%$ erythroid series with precursors in all of their maturational stages; $85 \%$ myeloid series and presence of ring-shaped neutrophils. The manual count was as follows: blastos (1\%), myelocytes (3\%), metamyelocytes (20\%), bands (13.5\%), neutrophils (48\%), pronormoblasts (14\%), histiocytes (0.5\%). Day 9: persistence of the pancytopenia (severe hypochromic microcytic anemia with $\mathrm{Hgb} 7.5 \mathrm{~g} / \mathrm{dl}$, moderate thrombocytopenia with platelets 71 thousand $/ \mathrm{mm}^{3}$, and grade II neutropenia with total neutrophils 1.93 thousand $/ \mathrm{mm}^{3}$ ). The MMF was suspended and the patient was given a blood transfusion of packed red blood cells. Day 18: Seventy-two hours without fever, the amphotericin B was suspended (accumulated dose of 1.05g), and itraconazolewas initiated at a dose of $100 \mathrm{mg}$ every 12 hours. The VGC was suspended due to absence of CMV viral load, as shown by quantitative PCR. Day 21: The patient remained afebrile, but with persistent anemia $(\mathrm{Hgb} 6.7 \mathrm{~g} / \mathrm{dl})$, and severe thrombocytopenia ( 6 thousand $/ \mathrm{mm}^{3}$ ). Treatment continued with transfusion of plateletpheresis and packed red blood cells. Granulocyte-Colony Stimulating Factor (G-CSF) was initiated for neutrophils of $0.83 \mathrm{ml} / \mathrm{mm}^{3}$, at a dose of $300 \mathrm{mcg} /$ day for 3 days. Day 22: Thrombopoietin-receptor agonist was initiated (Eltrombopag) at 50mg/day; and, on Day 26: 1000 platelets/mm [3] were reported with no evidence of bleeding, which led to continuance of the Eltrombopag and plateletpheresis. Day 30: Reports of sub-optimal levels of TAC $(<2 \mathrm{ng} / \mathrm{ml})$, probably the effect of rifampicin in the cytochrome $\mathrm{P} 450$ induction, and so the dose was incremented to 6mg/day. Day 31: Reports of Histoplasma sp. in myelocyte cultures (Figure 1). The DOTBAL was suspended and the itraconazole was maintained at a dose of $200 \mathrm{mg}$ every 12 hours. Day 34: Patient was afebrile, with persistent severe thrombocytopenia and no evidence of bleeding. The plateletpheresis continued and IV Immunoglobulin (IVIG) was initiated at $0.5 \mathrm{~g} / \mathrm{kg} /$ day for 3 days, and 48 hours later he presented with a progressive increase in platelets (61 thousand $/ \mathrm{mm}$ [3]) (Figure 2).

\section{Discussion}

We report the case of a male RTR who presented with clinical symptoms of the development of fever for 4 weeks and thrombocytopenia with progressive evolvement in severity. In over-immunosuppressed RTR with fever and pancytopenia, infectious processes always offer diagnostic possibilities; therefore, the opportune, empirical management of infections was initiated. In Mexico and Central America, HP is the most common mycosis, estimated to have an incidence of 0.1 to 0.29 cases $/ 100,000$ inhabitants [9]. The Human Immunodeficiency Virus (HIV) and immunosuppressors are associated with an increase in cases of HP $[5,10]$. In the present case, one possible factor that contributed to the development of DH was the over-immunosuppression (supraoptimal TAC levels). Assi et al. [3], published a retrospective study that included 152 cases of HP in SOT: in the first year, 34\% of cases were recorded and $81 \%$ of them corresponded to $\mathrm{DH}$; while effects on MO were recorded as mild to moderate in $21 \%$ and severe in $37 \%$; with no description on the level of the effect on cell lines [3]. The primary mechanisms for the development of HP in SOT are: 1) primary infection by inhalation; 2) reactivation of a previous infection due to over-immunosuppression; and, 3) infection derived from the donor, transmitted through the allograft [2]. In our patient, the

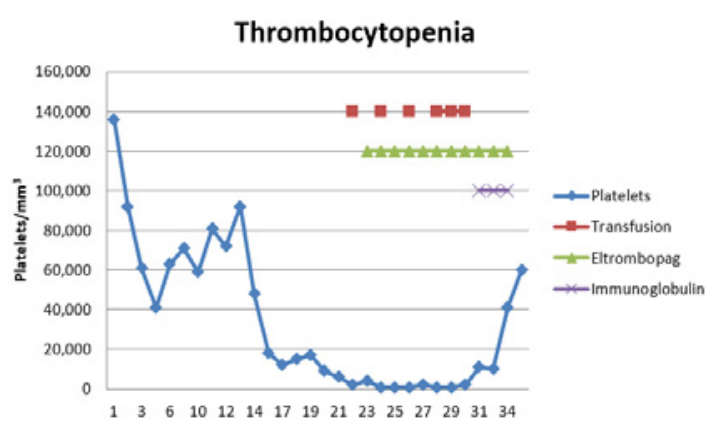

Figure 2: Evolution of thrombocytopenia. 
Table 1: Biochemical Characteristics.

\begin{tabular}{|c|c|c|c|c|c|c|c|c|c|c|c|c|c|c|c|c|}
\hline Test/Days & 1 & 3 & 5 & 8 & 9 & 12 & 14 & 16 & 17 & 21 & 23 & 28 & 29 & 32 & 35 & 36 \\
\hline Hemoglobin (gr/dl) & 11 & 7.9 & 7.7 & 7.8 & 7.2 & 8.5 & 8.1 & 7.5 & 7.2 & 6.7 & 8.5 & 7.7 & 7 & 7.5 & 7.2 & 8.3 \\
\hline Leucocytes (cells $/ \mathrm{mm}^{3}$ ) & 4 & 2.4 & 3.4 & 3.4 & 2.7 & 3.4 & 3.4 & 4 & 3.2 & 2.1 & 7.4 & 8.6 & 8 & 7 & 7 & 6.7 \\
\hline Neutrophils (cells $/ \mathrm{mm}^{[3]}$ ) & 3.14 & 1.98 & 2.89 & 2.6 & 1.9 & 2.2 & 1.7 & 1.6 & 1.4 & 0.8 & 5.3 & 3.08 & 3.1 & 2.9 & 2.3 & 2.3 \\
\hline Linfocites (cells/mm³) & 0.5 & 0.3 & 0.27 & 0.4 & 0.4 & 0.8 & 1.3 & 1.1 & 1.4 & 1 & 1.3 & 1.65 & 1.7 & 2.18 & 3.4 & 2.9 \\
\hline Platelets (cells/mm³) & 136 & 61 & 46 & 69 & 71 & 71 & 48 & 18 & 12 & 6 & 4 & 1 & 1 & 11 & 41 & 60 \\
\hline Procalcitonine (ng/ml) & 3.4 & - & 5.3 & - & 7.7 & 6.4 & - & - & 15 & - & 1.1 & - & - & 0.93 & - & - \\
\hline Urea (mg/dl) & 96 & 66 & 30 & 47 & 42 & 30 & 36 & 42 & 42 & 36 & 20 & - & 30 & 30 & 30 & 30 \\
\hline Creatinine (mg/dl) & 3.1 & 2 & 1.3 & 2.8 & 2.6 & 2.5 & 2.5 & 2.5 & 2.4 & 2.4 & 1.6 & - & 1.6 & 1.7 & 1.7 & 1.5 \\
\hline LDH U/I & 570 & - & - & - & - & - & - & - & - & - & - & - & - & - & - & - \\
\hline PT (sec) & - & - & $15 / 10.9$ & - & - & $16 / 11.2$ & - & $15 / 11.5$ & - & $16 / 11.3$ & $13 / 11.5$ & $13 / 11.5$ & - & - & $12 / 11.5$ & - \\
\hline INR & - & - & 1.33 & - & - & 1.45 & - & 1.27 & - & 1.43 & 1.08 & 1.14 & - & - & 1.06 & - \\
\hline PTT (sec) & - & - & $31 / 28.1$ & - & - & $33 / 27.5$ & - & $36 / 31.4$ & - & $42 / 29.4$ & $29 / 31.4$ & $39 / 28.5$ & - & - & $30 / 28.8$ & - \\
\hline Fibrinógeno (mg/dl) & - & - & $248 / 280$ & - & - & - & - & - & - & - & - & $292 / 304$ & - & - & - & - \\
\hline D-Dimer (ng/ml) & - & - & $>10,000$ & - & - & - & - & - & - & 1,431 & - & 768 & - & - & - & - \\
\hline Tacrolimus (ng/ml) & 17.6 & - & - & - & - & $<2$ & - & - & - & - & - & - & $<2$ & - & - & - \\
\hline
\end{tabular}

LDH: Lactate Dehydrogenase; INR: Internacional Normalized Ratio; PT: Prothrombin Time; PTT: Partial Thromboplastin Time

second option is the probable cause since he was exposed to bats on a trip four years prior to renal transplantation. The microorganism can remain for prolonged periods in the tissues after a primary infection, and has the capacity to be reactivated in immunosuppression [4]. In SOT, the signs and symptoms are unspecific and the majority include fever (>90\%), dry cough, generalized discomfort, and fatigue. In DH, fever is persistent, weight loss occurs, and also fatigue; and, in 25$60 \%$ of cases there is hepatomegaly and splenomegaly. Anemia and leukopenia are commonly reported [4]. However, there are no reports of thrombocytopenia in RTR. In 2017, Kutkut I. et al [6], reported a case of severe refractory thrombocytopenia with $\mathrm{DH}$, which, during the course of the illness, required multiple transfusions of platelets and IVIG at a dose of $1 \mathrm{~g} / \mathrm{kg} /$ day for two days without improvement. In this case, after the administration of amphotericin B liposomal at $3 \mathrm{mg} / \mathrm{kg} /$ day for 14 days, followed by itraconazole at $200 \mathrm{mg}$ /day for one year, it favored recuperation and normalization of the platelets, associated with an absence of the urinary antigen of H. capsulatum [6]. Contrary to the aforementioned case, our patient had platelet recuperation 48 hours after the application of the IVIG. The possible mechanisms by which $H$. capsulatum produces thrombocytopenia have been described: 1) by infiltration of MO affecting platelet production; 2) promoting platelet aggregation; or, 3) inducing ITP [6]. In the MO aspirate of our case, megakaryocytes producers of platelets were reported, and there were no yeasts of $H$. capsulatum found. Although one possibility is infiltration of $\mathrm{MO}$ as a cause of thrombocytopenia, our patient presented with severe refractory thrombocytopenia two weeks after the use of amphotericin B, with a significant response after the use of IVIG; thus, the probability exists that the cause was ITP induced by HP. In states of immunosuppression, Hood et al. [11], reported two cases of $\mathrm{DH}$ and thrombocytopenia. The first corresponded to a female kidney donor with severe thrombocytopenic purpura and splenomegaly, with the use of PDN $20 \mathrm{mg} /$ day and a fatal outcome due to intra-cerebral hemorrhage (the histoplasmin skin test, blood cultures, myelocyte cultures, and $\mathrm{MO}$ were negative for $H$. capsulatum). The second case corresponded to the RTR of the kidney donor from the first case: the immunosuppression documented was with azathioprine and PDN. At the $5^{\text {th }}$ post-transplant day, the patient presented with acute rejection and later with ITP and death. In both cases, the diagnosis of DH was documented in autopsy [11]. As possible mechanisms for thrombocytopenia, invasion of the MO by $H$. capsulatum, drug toxicity, activation of a latent ITP due to HP; formation of antibodies against platelets, production of a thrombocytopenic factor by $H$. capsulatum, and hypersplenism have been documented [11]. The present case agrees with the report by Hood et al. [11] with the development of ITP acquired by HP. The diagnosis of ITP is by exclusion, but the possible causes will always be infections. The morbidity-mortality is associated with hemorrhages and the first-line management is corticosteroids, with a response rate of $80 \%$. The IVIG is used to maintain platelet levels, and to favor decreases inthe doses of corticosteroids [12]. Kutkutet al. [6], showed recovery of $\mathrm{DH}$ at two months from the onset of antifungal treatment, and platelet normalization. On the other hand, Armitage et al. [13], reported two cases of DH and thrombocytopenia: the first case without improvement with the use of amphotericin B but with transitory improvement using corticosteroids, and complete recovery after splenectomy. The second case had transitory improvement to corticosteroids, with no response to splenectomy and a fatal outcome [13]. These cases correspond to immunocompetent patients; thus, the use of corticosteroids and/or splenectomy in the immunosuppressed could complicate DH. Therefore, the second line management for ITP (IVIG, thrombopoietin-receptor agonist "Eltrombopag" and splenectomy) could be a therapeutic option [14]. We did not use corticoids due to the risk of dissemination of the HP, and management was with platelet transfusions, Eltrombopag and, finally, IVIG with an adequate response. The use of TAC and MMF are associated to HP severity and mortality, so an early therapeutic measure was made in adjustment of the immunosuppression (MMF suspension and reduction of TAC) [15]. 


\section{Conclusion}

The clinical presentation of HP varies, which makes diagnosis and opportune treatment difficult. We present an atypical case of DH in a RTR with refractory thrombocytopenia, which, based on its clinical characteristics, we consider to be ITP induced by HP. This is the first case reported in our setting, and it opens up the possibility of new therapeutic strategies for thrombocytopenia induced by HP in immunocompromised RTRs.

\section{Informed Consent}

Written informed consent was obtained from the patient.

\section{References}

1. Tiwari SA, Ojha SS. Disseminated histoplasmosis diagnosed on bone marrow aspiration in a case of fever with thrombocytopenia. J Global Infect Dis. 2018; 10: 112-113.

2. Angsutararux $\mathrm{T}$, Chongtrakool $\mathrm{P}$, Sukpanichnant $\mathrm{S}$, Wongwaipijarn $\mathrm{K}$, Wangchinda W. Disseminated histoplasmosis in a kidney transplant patient. Transpl Infect Dis. 2021.

3. Assi M, Martin S, Wheat LJ, Hage C, Freifeld A, Avery R, et al. Histoplasmosis after solid organ transplant. Clin Infect Dis. 2013; 57: 1542-1549.

4. Freifeld AG, Wheat LJ, Kaul DR. Histoplasmosis in solid organ transplan recipients: Early diagnosis and treatment. Curr Opin Organ Transplant. 2009 14: 601-605

5. Wheat LJ, Azar MM, Bahr NC, Spec A, Relich RF, Hage C. Histoplasmosis. Infect Dis Clin North Am. 2016; 30: 207-227.

6. Kutkut I, Vater L, Goldman M, Czader M, Swenberg J, Fulkerson Z, et al Thrombocytopenia and disseminated histoplasmosis in immunocompetent adults. Clin Case Reports. 2017; 5: 1954-1960.
7. Nieto-Ríos JF, Serna-Higuita LM, Guzman-Luna CE, Ocampo-Kohn C Aristizabal-Alzate A, Ramírez I, et al. Histoplasmosis in renal transplant patients in an endemic area at a Reference Hospital in Medellin, Colombia. Transplant Proc. 2014; 46: 3004-3009.

8. Ling Q, Zhu W, Lu Q, Jin T, Ding S. Disseminated histoplasmosis in an immunocompetent patient from an endemic area: A case report. Med (United States). 2018; 97: 0-4.

9. Gómez BL. Histoplasmosis: Epidemiology in Latin America. Curr Fungal Infect Rep. 2011; 5: 199-205.

10. Sethi P, Treece J, Onweni C, Pai V, Arikapudi S, Kallur L, et al. Acute Kidney Injury, Immune Thrombocytopenic Purpura, and the Infection That Binds Them Together: Disseminated Histoplasmosis. J Investig Med High Impact Case Reports. 2017; 5.

11. Hood AB, Inglis FG, Lowenstein L, Dossetor JB, MacLean LD. Histoplasmosis and thrombocytopenic purpura: transmission by renal homotransplantation. Can Med Assoc J. 1965; 93: 587-592.

12. Onisâi $M$, Vlădăreanu $A M$, Spînu $A$, Găman $M$, Bumbea $H$. Idiopathic Thrombocytopenic Purpura (ITP) - new era for an old disease. Rom J Intern Med. 2019; 57: 273-283.

13. Armitage JO, Sheets RF. Idiopathic Thrombocytopenic Purpura in Patients With Histoplasmosis. JAMA J Am Med Assoc. 1977; 237: 2323-2324.

14. Cheng G, Saleh MN, Marcher C, Vasey S, Mayer B, Aivado M, et al Eltrombopag for management of chronic immune thrombocytopenia (RAISE): A 6-month, randomised, phase 3 study. Lancet. 2011; 377: 393-402.

15. Gajurel K, Dhakal R, Deresinski S. Diagnosis and treatment of histoplasmosis in solid organ transplant patients. Curr Opin Infect Dis. 2018; 31: 301-308. 